Revue internationale P.M.E.

Économie et gestion de la petite et moyenne entreprise

Revue

internationale

PME

\title{
Agressivité concurrentielle, taille de l'entreprise et performance
}

\section{Frédéric Le Roy}

Volume 14, numéro 2, 2001

URI : https://id.erudit.org/iderudit/1008692ar

DOI : https://doi.org/10.7202/1008692ar

Aller au sommaire du numéro

Éditeur(s)

Presses de l’Université du Québec

ISSN

0776-5436 (imprimé)

1918-9699 (numérique)

Découvrir la revue

Citer cet article

Le Roy, F. (2001). Agressivité concurrentielle, taille de l'entreprise et performance. Revue internationale P.M.E., 14(2), 67-84.

https://doi.org/10.7202/1008692ar

\section{Résumé de l'article}

Dans de nombreuses études récentes, centrées sur les grandes entreprises, il est considéré que les entreprises les plus performantes sont celles qui se comportent de façon agressive avec leurs concurrents. L'objet de cette recherche est d'évaluer si cette proposition normative est valable quelle que soit la taille de l'entreprise. Plusieurs hypothèses de recherche sont proposées et testées sur un échantillon de 105 entreprises industrielles. Le résultat le plus important de l'étude empirique est le suivant : pour les entreprises de petite taille, plus l'agressivité des concurrents est forte et plus l'agressivité de l'entreprise est forte, plus les performances sont faibles. 


\title{
Agressivité concurrentielle, taille de l'entreprise et performance
}

Frédéric LE ROY

ERFI, Université de Montpellier I

\section{MOTS CLÉS}

\section{Agressivité - Taille - Performance - PME Affrontement - Évitement}

\begin{abstract}
RÉSUMÉ
Dans de nombreuses études récentes, centrées sur les grandes entreprises, il est considéré que les entreprises les plus performantes sont celles qui se comportent de façon agressive avec leurs concurrents. L'objet de cette recherche est d'évaluer si cette proposition normative est valable quelle que soit la taille de l'entreprise. Plusieurs hypothèses de recherche sont proposées et testées sur un échantillon de 105 entreprises industrielles. Le résultat le plus important de l'étude empirique est le suivant: pour les entreprises de petite taille, plus l'agressivité des concurrents est forte et plus l'agressivité de l'entreprise est forte, plus les performances sont faibles.
\end{abstract}

\section{L'AUTEUR}

FRÉDÉRIC LE ROY est professeur en sciences de gestion. II travaille présentement au sein de l'Équipe de recherche sur la firme et l'industrie (ERFI) de l'Université de Montpellier I. Ses recherches ont, par le passé, porté sur le transfert de la stratégie militaire au management stratégique des entreprises, elles portent maintenant sur la dynamique de la concurrence. II a présenté plusieurs communications sur ces sujets de recherche dans de nombreux congrès francophones et anglophones et a publié dans diverses revues (Revue française de gestion, Revue finance contrôle stratégie, Annales des mines, Gérer et comprendre, etc.). II a aussi publié un ouvrage aux éditions Economica : Stratégie militaire et management stratégique des entreprises : une autre approche de la concurrence. Adresse : ERFI, Université de Montpellier I, ISEM, avenue de la Mer, B.P. 9659, 34054 Montpellier Cedex 1, France. Tél. : 0467506103. Courriel : <fred.leroy@libertysurf.fr>. 


\begin{abstract}
In many past research works focused on big companies, the most valuable firms appear to be those showing more aggressiveness toward their rivals. The object of this research is to evaluate the relevance of this normative proposition whatever the firm size. Several research hypothesis are proposed and tested on a sample of 105 industrial firms. The main result of the empirical study is that, for the small-sized firms, the higher the rivals' aggressiveness and the higher the firm's aggressiveness, the lower the firm's results.
\end{abstract}

\title{
RESUMEN
}

En numerosos estudios recientes, centrados en las empresas grandes, esto es considerado que las empresas màs acertadas son las que se comportan de un modo agresivo con sus competidores. El objeto de esta invetigación para estimar si esta proposición normativa es valida sobre el tamano de la empresa. Proponen a varias hipotesis de investigación y probados sobre una muestra de 105 preocupaciones industriales. El resultado mas importante del estudio empirico es el siguiente : para las empresas de pequeno tamano, mas la agressividad de los competidores es fuerte y mas la agresividad de la empresa es fuerte, mas los resultados son debiles.

\section{ZUSAMMENFASSUNG}

In verschiedenen kürzlich erschienen Studien über grosse Unternehmen, ist aufgezeigt worden, dass die leistungsstärksten Unternehmen diejenigen sind, die sich agressiv gegenüber ihren Konkurrenten verhalten. Ziel dieser Untersuchung ist zu beurteilen, ob diese normative Tatsache unabhängig ist von der Grösse der Unternehmen. Auf der Grundlage einer Stichprobe von 105 industriellen Unternehmen wurden verschiedene Forschungshypothesen vorgeschlagen und getestet. Das wichtigste Resultat dieser empirischen Studie für Unternehmen von kleiner Grösse kann folgendermassen zusammengefasst werden: Umso agressiver sich die Konkurrenz verhält, umso agressiver verhält sich das Unternehmen, desto schwächer sind die Unternehmensleistungen.

\section{Introduction}

Dans plusieurs recherches récentes, on a constaté que la plupart des secteurs sont le théâtre d'affrontements concurrentiels de plus en plus violents (Brahn, 1995 ; Brandemeyer et Schmidt, 1995 ; D’Aveni, 1995 ; Garda et Marn, 1994 ; Hinthorne, 1996 ; Potter, 1994). Certains auteurs affirment que ces affrontements n'ont d'autres résultats que la dégradation générale des marges et recommandent aux entreprises d'éviter de provoquer leurs concurrents (Brandemeyer et Schmidt, 1995 ; Garda et Marn, 1994 ; Porter, 1982). Inversement, d'autres auteurs considèrent que les marchés évoluent vers une nouvelle forme de concurrence, «l'hypercompétition », dans laquelle les entreprises les plus performantes ne sont pas celles qui évitent 
l'affrontement, mais celles qui introduisent les facteurs de déstabilisation du secteur, celles qui sont les plus agressives à l'égard de leurs concurrents (Craig, 1996; D’Aveni, 1995 ; Young, Smith et Grimm, 1996).

Peut-on alors considérer que ces propos normatifs qui affirment la nécessité d'un comportement agressif sont valables pour toutes les tailles d'entreprises? Doit-on, au contraire, conformément à une vision plus traditionnelle, continuer à considérer que les entreprises de petite taille ont davantage intérêt à éviter l'affrontement (Broom, Longenecker et Moore, 1983; Cohn et Lindberg, 1973; Kotler, 1988 ; McMillan, 1980 ; Porter, 1982) ? Pour répondre à ces questions, nous proposons, dans cette recherche, une première étude empirique des liens entre la taille, l'agressivité concurrentielle et les performances. Dans cette perspective, nous présenterons successivement les fondements théoriques de la recherche, la méthodologie, les résultats obtenus et leur interprétation.

\section{Fondements théoriques}

\subsection{Une nouvelle conception de la concurrence}

Dans les recherches antérieures, la concurrence a été analysée soit comme un ensemble de structures, soit comme un ensemble de décisions (Jacquemin, 1985). Dans le premier courant de pensée, les décisions des firmes sont contraintes par les structures des secteurs dans lesquels elles exercent leurs activités. Ces structures sont elles-mêmes déterminées par des conditions de base qui sont indépendantes des comportements des entreprises. Cette conception, issue de l'économie industrielle des origines, trouve de nombreux prolongements en sciences de gestion, que ce soit dans un transfert direct (Cool, Dierickx et Jemison, 1989; Porter, 1982) ou dans une approche plus indirecte qui fait de la décision stratégique la capacité à s'adapter à son environnement (Ansoff et McDonnell, 1990).

Le second courant de pensée trouve son origine dans l'école autrichienne fondée par Menger, Hayek et Von Mises (Young et al., 1996) et ses principaux prolongements dans les modélisations proposées par la «nouvelle économie industrielle » (Scherrer, 1980; Tirole, 1988) ainsi que dans des travaux récents en sciences de gestion (Chen, Smith et Grimm, 1992 ; Craig, 1996 ; D'Aveni, 1995 ; Guillotreau et Le Roy, 1999; Le Roy, 1998 ; Smith, Grimm et Gannon, 1993 ; Young et al., 1996). Dans ces recherches, il ne s'agit plus de caractériser les déterminants structurels de la concurrence, mais de prendre pour objet d'analyse les manœuvres concurrentielles des firmes. Les comportements des entreprises sont considérés comme relatifs à ceux de leurs principaux rivaux (Porac et al., 1995) et leurs performances dépendent de la dynamique de la rivalité concurrentielle, c'est-à-dire de l'ensemble des actions et réactions des concurrents directs.

Revue internationale P.M.E., vol. 14, nº 2, 2001

(C) 2001 - Presses de l'Université du Québec

Édifice Le Delta I, 2875, boul. Laurier, bureau 450, Sainte-Foy, Québec G1V 2M2 • Tél. : (418) 657-4399 - www.puq.uquebec.ca

Tiré de : Revue internationale P.M.E., vol. $14, \mathrm{n}^{\circ}$ 2, sous la direction de Pierre-André Julien. 
Dans cette perspective, certains auteurs considèrent que les comportements agressifs ont pour résultat essentiel la dégradation durable des marges dans un secteur (Brandemeyer et Schmidt, 1995 ; Garda et Marn, 1994 ; Porter, 1982). Les «guerres de prix » sont dénoncées comme le résultat d'un mauvais calcul de la part des firmes agressives (Brandemeyer et Schmidt, 1995 ; Porter, 1982) ou comme le résultat d'une paranoïa des concurrents (Garda et Marn, 1994). Inversement, pour d'autres auteurs, «l'hypercompétition» serait une évolution récente des marchés dans laquelle l'avantage concurrentiel dépendrait de la rapidité des actions concurrentielles (Young et al., 1996). Les entreprises qui ne chercheraient pas délibérément à déstabiliser leurs concurrents devraient, elles-mêmes, être l'objet de manœuvres agressives et, donc, connaître de graves difficultés. Il est alors recommandé d'être le premier à se livrer à des manœuvres agressives (D'Aveni, 1995), ce qui conduit à s'interroger sur le concept d'agressivité concurrentielle.

\subsection{Définitions de l'agressivité concurrentielle}

Le concept d'agressivité concurrentielle a été introduit relativement récemment en management stratégique. Dans la typologie proposée par Miles et Snow (1978), ce sont les «prospecteurs » qui font preuve de la plus grande agressivité dans leur recherche continuelle de nouvelles occasions d'affaires. Cette agressivité est donc comprise comme un comportement d'anticipation par rapport à des évolutions de la demande. Cette conception de l'agressivité est également celle de Fombrum et Ginsberg (1990), lorsqu'ils la définissent par l'ampleur de l'engagement des ressources et par le risque associé à l'allocation des ressources. Elle est alors mesurée par des indicateurs comme le pourcentage de R-D dans le chiffre d'affaires (CA). On retrouve une acception et des mesures très proches dans des recherches menées par Brockhoff et Pearson (1992), Calantone, Di Benedetto et Bhoovaraghavan (1994), Covin et Covin (1990) ou Zhara et Covin (1993) : les firmes «agressives » sont celles qui prennent les initiatives, qui introduisent les nouveaux produits, les nouvelles techniques de production, etc., alors que les firmes «passives» se contentent d'imiter les nouveaux produits, les nouvelles techniques, etc.

Dans les recherches portant sur la dynamique de la rivalité concurrentielle, l'agressivité concurrentielle n'est pas comprise comme un comportement relatif à la demande mais comme un comportement relatif à la concurrence. Ainsi, une entrée sur le marché peut être considérée comme plus ou moins agressive pour les concurrents en place (Williams, Tsa et Day, 1991). De même, la réaction des firmes en place à l'entrée d'un nouveau concurrent peut être plus ou moins agressive (Robinson, 1988). De façon plus générale, dans la dynamique de la concurrence, les actions et réactions concurrentielles peuvent être plus ou moins menaçantes et dommageables pour un concurrent (Chen et al., 1992). De façon plus particulière, plus une action affecte les positions d'un concurrent, plus elle est considérée comme agressive (Chen et al., 1992).

Revue internationale P.M.E., vol. 14, n 2, 2001 
Le concept d'agressivité concurrentielle est donc employé pour rendre compte soit d'un comportement relatif à la demande, soit d'un comportement relatif aux concurrents. Lumpkin et Dess (1996) proposent alors de distinguer clairement ces deux comportements et d'employer le concept de proactivité pour rendre compte d'un comportement d'anticipation par rapport à la demande de marché et de réserver le concept d'agressivité aux relations d'opposition concurrentielle. En suivant cette proposition de Lumpkin et Dess (1996), un comportement agressif sera défini comme une manœuvre qui affecte directement un concurrent.

\subsection{Hypothèses de recherche}

La définition de l'agressivité concurrentielle retenue dans cette recherche a été initialement introduite dans le cadre du respect du droit de la concurrence (Gundlach, 1990 ; Ordover et Saloner, 1989). Précisément, la question posée par les pouvoirs publics était la suivante: certaines entreprises fixent-elles des prix inférieurs à leurs coûts de production pendant une courte période afin, après avoir provoqué ainsi la sortie de leurs concurrents, de bénéficier des rentes de situation de monopole ? L'enjeu de cette interrogation était alors l'intervention des pouvoirs publics pour la protection de l'intérêt du consommateur (Williamson, 1977).

Cet enjeu a conduit de nombreux économistes à étudier le problème des «pratiques prédatrices ». Les recherches se sont centrées sur la question suivante : est-il rationnel, pour une entreprise, d'avoir l'intention d'éliminer ses rivaux (Gundlach, 1990) ${ }^{1}$ ? Les réponses à cette question ont été si nombreuses et si différentes qu'il n'est pas possible de les présenter toutes ${ }^{2}$. Nous développerons ici la théorie la plus ancienne et la plus discutée : la théorie des «poches profondes » (Ordover et Saloner, 1989). Selon cette théorie, introduite par Edwards en 1955, une grande entreprise détient un pouvoir spécifique du simple fait de sa taille, puisqu'elle lui permet d'assumer à plus long terme des pertes financières qu'une entreprise de petite taille (Ordover et Saloner, 1989).

Cette argumentation intuitive à l'origine a fait l'objet de nombreux développements théoriques, dont l'un des plus récents est proposé par Fundenberg et Tirole (1986). Pour ces deux auteurs, s'il est fait l'hypothèse d'une symétrie des ressources financières, c'est-à-dire s'il est considéré que les conditions d'accès aux ressources externes (par l'emprunt) sont les mêmes que les conditions d'accès

1. En effet, s'il est rationnel de tenter des manœuvres uniquement fondées sur une intention d'élimination et si les dirigeants sont bien rationnels, il est nécessaire d'intervenir pour éviter la constitution de monopoles. En revanche, s'il est irrationnel de tenter d'éliminer ses concurrents, les pouvoirs publics ne doivent pas intervenir.

2. Pour une revue des travaux antérieurs voir Ordover et Willig (1981) et Ordover et Saloner (1989), Scherrer (1980), Scherrer et Ross (1990) et Tirole (1988).

Revue internationale P.M.E., vol. 14, n 2, 2001

(C) 2001 - Presses de l'Université du Québec

Édifice Le Delta I, 2875, boul. Laurier, bureau 450, Sainte-Foy, Québec G1V 2M2 • Tél. : (418) 657-4399 - www.puq.uquebec.ca

Tiré de : Revue internationale P.M.E., vol. 14, $\mathrm{n}^{\circ} 2$, sous la direction de Pierre-André Julien. 
aux ressources internes, une entreprise objet d'une pratique prédatrice pourra emprunter jusqu'à ce que le «prédateur » arrive à la limite de son seuil d'épuisement des ressources internes. La prédation devient donc un comportement irrationnel.

En revanche, s'il est fait l'hypothèse d'une asymétrie des ressources financières à l'avantage des ressources internes par rapport aux ressources externes (à cause des imperfections du marché du crédit), le fait d'avoir des "poches profondes », c'est-à-dire des ressources internes de financement supérieures à celles des concurrents, permet la réussite d'une pratique prédatrice. En effet, dans ce cas, une entreprise qui dispose de ressources financières internes inférieures à celles de son prédateur ne pourra pas compenser infiniment ses pertes d'exploitation en cas de guerre des prix par un recours à l'emprunt et sera donc contrainte de fermer ou de vendre. Les entreprises de grande taille, qui disposent de ressources financières internes importantes, devraient donc être plus agressives que les entreprises de petite taille.

Inversement, toujours en suivant Fundenberg et Tirole (1986), il serait irrationnel pour une entreprise de petite taille d'agresser une entreprise plus grande. Cette proposition rejoint les travaux de nombreux auteurs qui s'accordent pour dire qu'une entreprise de petite taille ne peut lutter directement contre des entreprises plus grandes. La meilleure stratégie consiste alors à éviter l'affrontement direct en se différenciant et/ou en se centrant sur un segment de la clientèle peu ou mal occupé par les grandes entreprises (Broom et al., 1983; Cohn et Lindberg, 1973; Kotler, 1988 ; McMillan, 1980 ; Porter, 1982). Il est donc possible de supposer que plus une entreprise est petite, plus elle cherche à éviter l'affrontement et, donc, moins elle est agressive par rapport à ses concurrents.

\section{Proposition 1: L'agressivité concurrentielle est liée à la taille de l'entreprise.}

\section{H 1.1: Les entreprises de grande taille sont significativement plus agressives que} les entreprises de petite taille.

\section{H 1.2: Les entreprises de petite taille sont significativement plus agressées que les entreprises de grande taille.}

À cette première question sur le lien entre la taille d'une entreprise et sa propension à être agressive ou agressée s'ajoutent des interrogations sur les liens entre la taille d'une entreprise et sa propension à être agressive et agressée. Le lien entre l'agressivité des concurrents et l'agressivité de l'entreprise est-il plus fort pour une entreprise de grande taille que pour une entreprise de petite taille? En suivant Fundenberg et Tirole (1986), une entreprise de petite taille, objet d'une manœuvre agressive, n'a pas les moyens de financement internes qui lui permettraient de supporter l'affrontement. Il est donc possible de supposer qu'elle ne réagira pas 
agressivement à une manœuvre agressive et qu'elle tentera plutôt une manœuvre « proactive » (Miles et Snow, 1978) ou « entrepreneuriale (Julien et Marchesnay, 1989) à l'égard d'un segment de clientèle peu ou mal desservi. Inversement, les entreprises de grande taille ont les moyens de soutenir durablement une pression sur les prix (Fundenberg et Tirole, 1986) et ont intérêt à réagir rapidement et violemment à une manœuvre agressive de la part d'un concurrent pour éviter de perdre le leadership de leur secteur (Tirole, 1988).

\section{Proposition 2 : Le lien entre l'agressivité de l'entreprise et l'agressivité des concurrents est fonction de la taille de l'entreprise.}

H 2.1 : Pour les entreprises de petite taille, l'agressivité de l'entreprise et l'agressivité des concurrents ne sont pas significativement liées.

H 2.2: Pour les entreprises de grande taille, l'agressivité de l'entreprise et l'agressivité des concurrents sont significativement liées.

Après ces questions sur la propension à être agressif et / ou agressé, il convient de s'interroger sur le lien entre les comportements agressifs et les performances. Pour Fundenberg et Tirole (1986), les entreprises qui disposent de ressources internes limitées ne peuvent financièrement supporter un affrontement. De la même façon, dans de nombreuses recherches (Broom et al., 1983 ; Cohn et Lindberg, 1973 ; Kotler, 1988 ; McMillan, 1980 ; Porter, 1982), il est considéré qu'une entreprise de petite taille se place dans une situation d'évitement de l'affrontement essentiellement parce qu'elle n'a pas les moyens de soutenir financièrement une concurrence directe. Une forte agressivité des entreprises de petite taille devrait donc se traduire par une baisse de leurs performances.

Inversement, les entreprises de grande taille pourraient se placer en situation d'affrontement puisqu'elles ont les moyens de compenser une dégradation de leurs marges dans un secteur ou sur un marché par des ressources financières tirées de leurs autres activités (Fundenberg et Tirole, 1986 ; Levy, 1989). Une forte agressivité ne devrait donc pas entraîner de baisse de leurs performances.

\section{Proposition 3 : Les relations entre l'agressivité d'une entreprise et ses perfor- mances sont fonction de sa taille.}

H 3.1 : L'agressivité d'une entreprise de grande taille n'entraîne pas une baisse de ses performances.

H 3.2: L'agressivité d'une entreprise de petite taille entraîne une baisse de ses performances.

Revue internationale P.M.E., vol. 14, n 2, 2001

(C) 2001 - Presses de l'Université du Québec 
Proposition 4 : Les relations entre l'agressivité des concurrents et les performances sont fonction de la taille de l'entreprise agressée.

H 4.1 : L'agressivité des concurrents d'une entreprise de grande taille n'entraîne pas une baisse de ses performances.

H 4.2 : L'agressivité des concurrents d'une entreprise de petite taille entraîne une baisse de ses performances.

Ces tendances devraient être d'autant plus marquées qu'une petite entreprise réagit agressivement à des comportements agressifs. En effet, en réagissant d'une façon agressive à une agression, une petite entreprise se place dans une situation d'affrontement qu'elle n'a pas, a priori, les moyens financiers d'assumer (Fundenberg et Tirole, 1986 ; Scherrer, 1980; Tirole, 1988). Les petites entreprises qui réagissent agressivement à des manœuvres agressives devraient donc enregistrer une baisse de leurs performances, ce qui ne devrait pas être le cas des entreprises de grande taille.

Proposition 5 : La relation entre l'agressivité de la concurrence, l'agressivité de l'entreprise et les performances est fonction de la taille.

H 5.1: Pour les entreprises de petite taille, plus l'agressivité des concurrents est forte et plus l'agressivité de l'entreprise est forte, plus les performances sont faibles.

H 5.2: Pour les entreprises de grande taille, il n'y a pas de lien entre l'agressivité des concurrents, l'agressivité de l'entreprise et les performances.

\section{Méthodologie}

\section{1. Échantillon}

La collecte des données a été effectuée par des entretiens en face à face. Un questionnaire a été élaboré et administré (par des étudiants de la Faculté de sciences économiques de l'Université de Montpellier I) auprès de 190 hauts responsables d'entreprises industrielles (sélection à partir du Kompass régional). L'échantillon devait être constitué d'entreprises de toute taille (pour évaluer l'effet de la taille). Un certain nombre de questionnaires n'ont pas été utilisés pour plusieurs raisons : parce qu'ils étaient largement incomplets, parce que la personne interrogée n'était pas un des principaux décideurs de l'entreprise ou parce qu'ils étaient remplis de façon trop rapide (après compte rendu de l'étudiant). L'effectif global de l'échantillon a donc été ramené à 105 entreprises. Il s'agit d'un échantillon de convenance, au sens de Evrard, Pras et Roux (1993). 
TABLEAU 1

Présentation de l'échantillon

\begin{tabular}{lccc}
\hline & Indépendantes & Appartenant à un groupe & Total \\
\hline 10 à 49 salariés & 32 & 10 & 42 \\
50 à 200 salariés & 17 & 16 & 33 \\
200 à 500 salariés & 3 & 8 & 11 \\
+ de 500 salariés & 5 & 14 & 19 \\
Total & 57 & 48 & 105 \\
\hline
\end{tabular}

\subsection{Mesure des variables}

Selon le critère le plus courant (Julien, 1990), la taille de l'entreprise est définie par rapport au nombre de salariés. Les entreprises dont l'effectif est inférieur à 200 salariés seront considérées comme de petite taille. Toutefois, ne seront prises en compte dans cette sélection que les entreprises indépendantes sur le plan du capital. Les entreprises de moins de 200 salariés qui appartiennent à un groupe seront considérées comme des entreprises de grande taille. En effet, étant donné que l'asymétrie des modes de financement est à l'origine des hypothèses, il ne semble pas possible d'agréger des entreprises devant faire face seule à des phénomènes d'agression et des entreprises disposant d'apports de capitaux de groupes.

Les mesures de l'agressivité concurrentielle existantes (Ansoff et McDonnell, 1990 ; Fombrum et Ginsberg, 1990) correspondent à une définition de l'agressivité comme un comportement proactif (Lumpkin et Dess, 1996). Pour proposer une mesure de l'agressivité comme un comportement relatif à la concurrence, des concepts issus des études portant sur les conflits dans les canaux de distribution ont été utilisés (Brown et Day, 1981). Trois notions fondamentales ont été distingués ; la fréquence, l'intensité et l'importance du conflit, pour construire des échelles de mesure de l'agressivité des concurrents et de l'agressivité de l'entreprise (Le Roy, 1996) ${ }^{3}$.

L'échelle de mesure de l'agressivité de la concurrence comprend des énoncés relatifs à la fréquence des manœuvres agressives, au nombre de concurrents agressifs, à l'intensité des manœuvres agressives et à leur importance pour l'entreprise ${ }^{4}$. De même, l'échelle de mesure de l'agressivité de l'entreprise comprend des énoncés

3. Une manœuvre agressive est présentée au répondant comme un comportement (baisse de prix, démarchage, etc.) visant à prendre directement une partie de la clientèle de un ou plusieurs concurrents.

4. L'alpha de Cronbach est de 0,83 .

Revue internationale P.M.E., vol. 14, $\mathrm{n}^{\circ}$ 2, 2001 
relatifs à la fréquence des manœuvres agressives, au nombre de concurrents agressés, à l'intensité des manœuvres agressives et à leur importance pour les concurrents 5 . Les moyennes et les écarts types sont exposés dans le tableau 2.

TABLEAU 2

Moyennes et écart types pour l'agressivité

\begin{tabular}{lcccc}
\hline Concepts & Moy. & E.T. & Min. & Max. \\
\hline Man Agres Con & 3,81 & 0,94 & 1 & 5 \\
Man Agress Etps & 3,31 & 1,07 & 1 & 5 \\
\hline
\end{tabular}

Deux contraintes majeures ont orienté le choix d'une mesure des performances : la diversité sectorielle et la diversité des tailles d'entreprises. Tout d'abord, la diversité sectorielle rend discutable le choix d'une mesure «objective» de la performance (Chakravarthy, 1986). Ensuite, il semble difficile de comparer les performances des entreprises de grande taille et de petite taille selon ces même critères «objectifs», puisque, pour les entreprises de petite taille, les données objectives sont difficiles à obtenir auprès des dirigeants et peu disponibles dans le domaine public. De plus, ces données sont très difficiles à interpréter. Par exemple, de faibles profits dans une petite entreprise en croissance peuvent être dus à un très fort réinvestissement (Covin et Covin, 1990).

Pour maintenir une homogénéité de la mesure, les données subjectives, justifiées par Dess et Robinson (1984) et utilisées notamment par Covin et Covin (1990) et Gupta et Govindarajan (1984), apparaissent comme plus pertinentes. L'échelle de mesure sera donc constituée d'énoncés relatifs à la rentabilité actuelle de l'entreprise, à l'évolution de la rentabilité sur les trois dernières années, à l'évolution du CA sur les trois dernières années, à l'évolution de la part de marché sur les trois dernières années et à l'évolution de l'effectif sur les trois dernières années ${ }^{6}$.

\subsection{Méthode de traitement des données}

Les hypothèses 1.1 et 1.2 seront testées en comparant les moyennes des scores (des différentes dimensions de l'agressivité concurrentielle) obtenus dans les deux groupes (les entreprises de petite taille et les entreprises de grande taille). Les hypothèses 2.1 et 2.2 seront testées par une analyse de corrélation simple entre l'agressivité de la concurrence et l'agressivité de l'entreprise. Pour tester les hypothèses 3.1, 3.2, 4.1 et 4.2, l'analyse consistera en un corrélation simple entre

5. L'alpha de Cronbach est de 0,91 .

6. L'alpha de Cronbach est de 0,73 . 
les différentes dimensions de l'agressivité concurrentielle et les performances. Enfin, les hypothèses 5.1 et 5.2 seront testées à partir de l'analyse de la variable modératrice (Sharma, Durand et Gur-Arie, 1981) ${ }^{7}$.

\section{Résultats}

Le tableau 3 montre que l'hypothèse 1.1 est validée à un niveau faible $(\mathrm{p}<0,1)$ pour les manœuvres agressives de la part de l'entreprise et que l'hypothèse 1.2 n'est pas validée pour les manœuvres agressives de la part des concurrents. Les entreprises de grande taille ne sont donc pas plus à l'origine ou moins l'objet de manœuvres agressives que les entreprises de petite taille.

TABLEAU 3

Tests pour les hypothèses 1.1 et 1.2

\begin{tabular}{lccc}
\hline & Petite taille & Grande taille & Test \\
\hline Man Ag Conc & 3,78 & 3,82 & - \\
Man Ag Etps & 3,19 & 3,47 & $*$ \\
\hline
\end{tabular}

$* \mathrm{p}<0,1, * * \mathrm{p}<0,05$

Le tableau 4 montre que, pour les entreprises de petite taille, il y a une forte liaison entre les manœuvres agressives de la part de l'entreprise et les manœuvres agressives de la part des concurrents $(\mathrm{r}=0,41, \mathrm{p}<0,001)$. L'hypothèse 2.1 est donc réfutée : pour les entreprises de petite taille, l'agressivité de l'entreprise et l'agressivité des concurrents sont significativement liées. Le tableau 4 montre également que, pour les entreprises de grande taille, il y a une forte liaison entre les manœuvres agressives de la part de l'entreprise et les manœuvres agressives de la part des concurrents $(r=0,49, p<0,001)$. L'hypothèse 2.2 est donc validée.

\section{TABLEAU 4}

Tests pour les hypothèses 2.1 et 2.2

\begin{tabular}{ccc}
\hline & Entreprises de petite taille & Entreprises de grande taille \\
\hline & Man Ag Conc & Man $\mathrm{Ag}$ Conc \\
Man Ag Etps & $0,41 * * *$ & $0,49 * * *$ \\
\hline
\end{tabular}

$* \mathrm{p}<0,05, * * \mathrm{p}<0,01, * * * \mathrm{p}<0,001$

7. Cette analyse revient à distinguer trois variables : $\mathrm{X}$ la variable « prédictive» (predictor), $\mathrm{Y}$ la variable «critère» (criterion) et $\mathrm{Z}$ la variable «modératrice» (moderator). Elle consiste à poser l'équation suivante: $\mathrm{Y}=a+b 1 \mathrm{X}+b 2 \mathrm{Z}+b 3 \mathrm{X}$.Z. Pour que $\mathrm{Z}$ puisse être considérée comme une variable modératrice, il faut que $b 3$ soit différent de 0 .

Revue internationale P.M.E., vol. 14, nº 2, 2001 
Le tableau 5 montre que, pour les entreprises de grande taille, il n'y a pas de liaison entre les manœuvres agressives de la part de l'entreprise et les performances. L'hypothèse 3.1 est donc validée: l'agressivité d'une entreprise de grande taille n'entraîne pas une baisse de ses performances. Il montre en outre que, pour les entreprises de petite taille, la liaison entre les manœuvres agressives de la part de l'entreprise et les performances est faible $(r=-0,23, p<0,1)$. L'hypothèse 3.2 peut donc difficilement être acceptée comme validée : l'agressivité d'une entreprise de petite taille n'entraîne pas une baisse de ses performances.

TABLEAU 5

Tests pour les hypothèses 3.1, 3.2, 4.1 et 4.2

\begin{tabular}{lcc}
\hline $\begin{array}{l}\text { Corrélations entre l'agressivité de } \\
\text { l'entreprise et les performances }\end{array}$ & $\begin{array}{c}\text { Entreprises de } \\
\text { petite taille }\end{array}$ & $\begin{array}{c}\text { Entreprises de } \\
\text { grande taille }\end{array}$ \\
\hline Man Ag Etps & $-0,23 *$ & 0,14 \\
Man Ag Conc & $-0,26 *$ & 0,00 \\
\hline
\end{tabular}

$* \mathrm{p}<0,1$

Le tableau 5 indique qu'il n'y a pas de liaison entre les manœuvres agressives de la part des concurrents et les performances pour les entreprises de grande taille. L'hypothèse 4.1 est donc validée: l'agressivité des concurrents d'une entreprise de grande taille n'entraine pas une baisse de ses performances. En outre, on voit que, pour les entreprises de petite taille, il n'y a qu' une liaison faiblement significative entre les manœuvres agressives de la part des concurrents et les performances $(\mathrm{r}=-0,26 ; \mathrm{p}<0,1)$. L'hypothèse 4.2 peut donc difficilement être acceptée comme validée : l'agressivité des concurrents d'une entreprise de petite taille n'entraîne pas une baisse de ses performances.

Le tableau 6 montre que, pour les entreprises de petite taille, l'hypothèse 5.1 est vérifiée à un niveau important de signification $(\mathrm{b} 3=-0,19, \mathrm{p}<0,01)$ : plus une entreprise de petite taille est l'objet de manœuvres agressives de la part de ses concurrents et plus elle est agressive, plus ses performances sont faibles.

TABLEAU 6

Tests pour les hypothèses 5.1 et 5.2

\begin{tabular}{lcc}
\hline & \multicolumn{2}{c}{ Performances } \\
\hline Interaction & Etps pet taille & Etps gr taille \\
MAC * MAE & $-0,19 * *$ & 0,08 \\
\hline
\end{tabular}

$* * \mathrm{p}<0,01$ 
Dans le même tableau, on voit que l'hypothèse 5.2 est vérifiée pour les manœuvres agressives: il n'y a pas de lien entre l'agressivité des concurrents, l'agressivité de l'entreprise et les performances pour les entreprises de grande taille.

\section{Interprétation des résultats}

Les résultats obtenus après les tests des hypothèses 1.1 et 1.2 ne sont, dans l'ensemble, pas conformes aux résultats des recherches antérieures. En effet, alors que les entreprises de petite taille devraient être plus agressées que les entreprises de grande taille (Fundenberg et Tirole, 1986), elles ne sont pas plus l'objet de manœuvres agressives. De même, alors qu'elles devraient éviter elles-mêmes de déclencher des affrontements (Broom et al., 1983 ; Cohn et Lindberg, 1973 ; Kotler, 1988 ; McMillan, 1980 ; Porter, 1982), elles ne sont pas moins à l'origine de manœuvres agressives.

Les tests des hypothèses 2.1 et 2.2 ne sont pas non plus conformes aux travaux antérieurs. En effet, alors que les entreprises de petite taille devraient éviter de répondre agressivement à des manœuvres agressives, la relation entre les manœuvres agressives de la part des concurrents et les manœuvres agressives de la part de l'entreprise n'est pas fonction de la taille de l'entreprise (elles sont fortement liées quelle que soit la taille). La première conclusion importante de cette recherche est donc que, contrairement aux propositions des recherches antérieures, la taille de l'entreprise n'implique pas des comportements différents en termes d'agressivité concurrentielle.

Les tests des hypothèses 3.1 et 4.1 révèlent qu'il n'y a pas de liaison entre l'agressivité et les performances pour les entreprises de grande taille. De la même façon, il n'y a qu'une relation faiblement significative pour les entreprises de petite taille (hypothèses 3.2 et 4.2). Il n'est donc pas possible d'établir une liaison simple entre l'agressivité concurrentielle et les performances. Ce qui peut être interprété comme l'absence d'influence de la taille sur le lien entre l'agressivité concurrentielle et les performances.

Toutefois, si la taille n'a pas d'influence sur la relation entre le fait d'être agressif $o u$ agressé et les performances, le test des hypothèses 5.1 et 5.2 démontre qu'elle a une influence sur la relation entre le fait d'être agressif et agressé et les performances. En effet, plus les entreprises de petite taille se livrent à des manœuvres agressives et plus leurs concurrents se livrent à des manœuvres agressives aussi, plus leurs performances diminuent. Pour ces entreprises, plus que le fait d'être agressives ou d'être agressées, c'est la convergence de leur agressivité avec celle de leurs concurrents qui conduit à une baisse des performances.

Ces résultats contredisent les études portant sur le « lien stratégique (strategic fit), qui affirment que, pour être performante, une entreprise doit s'aligner sur les caractéristiques de son environnement (Ansoff et McDonnell, 1990 ; Venkataraman,

Revue internationale P.M.E., vol. 14, n 2, 2001

(C) 2001 - Presses de l'Université du Québec

Édifice Le Delta I, 2875, boul. Laurier, bureau 450, Sainte-Foy, Québec G1V 2M2 • Tél. : (418) 657-4399 - www.puq.uquebec.ca

Tiré de : Revue internationale P.M.E., vol. 14, $\mathrm{n}^{\circ} 2$, sous la direction de Pierre-André Julien. 
1989). Ils sont également contradictoires avec les travaux récents sur «l'hypercompétition » selon lesquels les entreprises ont intérêt à provoquer l'affrontement dans leur secteur d'activité (Craig, 1996; D'Aveni, 1995; Young et al., 1996). Manifestement, les dirigeants de PME n'ont aucun intérêt à imiter d'éventuels comportements agressifs de la part de leurs concurrents ou à tenter de déstabiliser leurs secteurs et ainsi provoquer des réactions agressives de la part de leurs concurrents.

Ces résultats concordent, en revanche, avec les recherches dont les auteurs soutiennent que les PME sont plus vulnérables que les grandes entreprises dans des situations d'affrontement. Si le modèle de Fundenberg et Tirole (1986) ne permet pas de prédire les comportements (pas de différence en termes d'agressivité concurrentielle selon la taille), il permet de prédire les résultats de ces comportements. En effet, conformément à ce modèle, lorsque les entreprises de petite taille entrent dans une situation de conflit avec leurs concurrents, elles sont bien plus affectées sur le plan des performances financières que les entreprises de grande taille. Elles ont donc un intérêt certain à suivre des stratégies d'évitement de la concurrence (Broom et al., 1983 ; Cohn et Lindberg, 1973 ; Kotler, 1988 ; McMillan, 1980 ; Porter, 1982).

Les résultats soulèvent la question suivante : pour quelle raison les dirigeants de PME se placent en situation d'affrontement s'ils n'ont pas les moyens de l'assumer ? Ce qui amène à se demander si une entreprise de petite taille peut constamment choisir d'être plus ou moins agressive par rapport à ses concurrents, si elle dispose d'une certaine latitude qui lui permettrait de ne pas se placer en situation d'affrontement. Plus précisément, en référence aux travaux sur les «choix stratégiques » (Lawless et Finch, 1989), il est possible de se demander si l'acceptation d'une situation d'affrontement résulte d'un choix du dirigeant, ce qui se révèle en l'occurrence une mauvaise décision, ou d'un déterminisme de l'environnement auquel le dirigeant de PME ne peut que se soumettre.

Dans le premier cas, il s'agirait d'un comportement irrationnel de la part du dirigeant de PME, dont il reste à établir les déterminants dans des recherches futures. Le rôle de l'affect dans les relations de concurrence pourrait ainsi être plus approfondi. Si l'on écarte l'hypothèse de l'irrationalité des dirigeants, les résultats autorisent à penser que, si l'évitement est bien le meilleur choix qui s'offre à une $\mathrm{PME}$, il est pour le moins difficile à réaliser. Autrement dit, ce n'est pas parce qu'un dirigeant de PME tente de suivre un stratégie d'évitement qu'il y parvient. Au contraire, il semblerait que les concurrents des entreprises de petite taille aient les moyens de les contraindre à l'affrontement alors qu'elles n'y ont pas du tout intérêt.

Il est alors possible de se demander si cette contrainte concurrentielle est la même pour toutes les PME. En effet, une entreprise de petite taille en concurrence avec des entreprises de taille comparable est sans doute moins contrainte à l'affron- 
tement que si elle est en situation de rivalité avec des entreprises de plus grande taille. Dans cette perspective, il s'agirait de compléter cette recherche par des travaux portant moins sur les effets de la taille absolue que sur ceux de la taille relative. En ce sens, ce serait bien la comparaison dyadique des ressources internes des acteurs en situation de rivalité qui serait déterminante pour expliquer leurs comportements.

En posant le problème de l'effet taille de façon relative, d'autres questions de recherches mériteraient d'être approfondies. Par exemple, nous avons supposé que les entreprises de petite taille appartenant à un groupe avaient accès à toutes les ressources financières internes de ce groupe. Lever cette hypothèse reviendrait à mieux analyser les comportements concurrentiels des PME appartenant à des groupes relativement au transfert de ressources effectuées par ces groupes. Il serait ainsi particulièrement stimulant d'observer un éventuel changement de comportement d'une PME en cas d'acquisition par une entreprise de plus grande taille. Dans le même ordre d'idées, il serait sans doute très intéressant d'analyser les rapprochements entre entreprises de petite taille, que ce soit sous la forme d'alliance ou de fusion-acquisitions, comme la possibilité de modifier, non pas leur taille absolue, mais bien leur taille relative dans une situation de plus forte agressivité concurrentielle.

\section{Conclusion}

L'objet de cette recherche était de proposer une première étude empirique des liens entre l'agressivité concurrentielle, la taille et les performances. Il apparaît, tout d'abord, que la taille de l'entreprise n'induit pas des comportements différents dans des contextes agressifs, que ce soit en termes de propension à l'agressivité ou en termes de liaison entre l'agressivité de l'entreprise et celle de ses concurrents. Il semble, ensuite, qu'il n'y ait pas de liaison, quelle que soit la taille de l'entreprise, entre l'agressivité de l'entreprise et l'agressivité des concurrents prises séparément et les performances. Enfin, le dernier résultat important est la baisse des performances que connaissent les petites entreprises lorsqu'il y a une convergence de leur propre agressivité avec celle de leurs concurrents.

Il convient, bien entendu, de n'accepter les résultats obtenus que dans le cadre des limites de l'étude, qui sont celles de toute étude empirique fondée sur une observation transversale d'un échantillon. Ces limites conduisent à une interprétation prudente des résultats et ouvrent de nombreuses pistes de recherches. Tout d'abord, il conviendrait d'introduire dans l'analyse les notions de choix stratégique et de déterminisme de l'environnement concurrentiel. Ensuite, tout un champ de recherche semble ouvert sur les choix qui s'offrent à un dirigeant de PME aux prises avec une situation d'affrontement concurrentiel. Enfin, il faudrait mieux tenir compte des effets non plus de la taille absolue mais de ceux de la taille relative.

Revue internationale P.M.E., vol. 14, n 2, 2001

(C) 2001 - Presses de l'Université du Québec

Édifice Le Delta I, 2875, boul. Laurier, bureau 450, Sainte-Foy, Québec G1V 2M2 • Tél. : (418) 657-4399 - www.puq.uquebec.ca

Tiré de : Revue internationale P.M.E., vol. $14, \mathrm{n}^{\circ}$ 2, sous la direction de Pierre-André Julien. 
En définitive, comme le recommandent Lumpkin et Dess (1996), les questions de l'agressivité concurrentielle et de sa gestion par un dirigeant de PME mériteraient davantage d'intérêt qu'une allusion parfois un peu trop rapide à une stratégie d'évitement (Broom et al., 1983 ; Cohn et Lindberg, 1973 ; Kotler, 1988 ; McMillan, 1980 ; Porter, 1982) ou à l'affirmation, tout aussi rapide, de la nécessité d'adopter un comportement agressif quelle que soit la taille de l'entreprise (Craig, 1996 ; D’Aveni, 1995 ; Young et al., 1996).

\section{Bibliographie}

Ansoff, I. et E. MCDonnell (1990), Implanting Strategic Management, New York, Prentice-Hall.

BRAHN, R. (1995), « National targeting policies, high-technology industries and excessive competition », Strategic Management Journal, vol. 16, numéro spécial, p. 71-91.

BRANDEMEYER, K. et M. SCHMIDT (1995), «La guerre des prix dans les parfums : de l'autodestruction d'un secteur », Décisions Marketing, $\mathrm{n}^{\circ}$ 6, p. 15-22.

BROCKHOFF, K. et A. PEARSON (1992), « Technical and marketing aggressiveness and the effectiveness of research and development », IEEE Transactions on Engineering Management, vol. 39, no 4, p. 318-324.

Broom, H.N., J.G. LongeneCKer et C.W. Moore (1983), Small Business Management, Cincinnati, $\mathrm{OH}$, Southwest Publishing.

BROWN, J.R. et R.L. DAY (1981), « Measures of manifest conflict in distribution channels », Journal of Marketing Research, vol. 18, nº 3, p. 263-274.

Calantone, R. J., C. A. Di Benedetto et S. Bhoovaraghavan (1994), «Examining the relationship between degree of innovation and new product success », Journal of Business Research, vol. 30, n 2, p. 143-148.

ChaKRAVARTHY, B.S. (1986), «Measuring strategic performance», Strategic Management Journal, vol. 7, $\mathrm{n}^{\circ}$ 5, p. 437-458.

Chen, M.-J., K.G. SMITH et C.M. GrimM (1992), «Action characteristics as predictor of competitive responses », Management Science, vol. 35, n 3, p. 439-455.

CoHn, T. et R.A. LindBerg (1973), How Management Is Different in Small Companies, New York, American Management Association.

COOL, K., I. DIERICKX et D. JEMINSON (1989), «Business strategy, market structure and risk-return relationships : a structural approach», Strategic Management Journal, vol. $10, n^{\circ} 6$, p. 507-522.

Covin, J.G et T.J. Covin (1990), «Competitive aggressiveness, environmental context, and small firm performance », Entrepreneurship Theory and Practice, vol. 14, $\mathrm{n}^{\circ} 4$, p. 35-49.

CRAIG, T. (1996), « The Japanese beer war : initiating and responding to hypercompetition in new product development », Organization Science, vol. 7, n 3, p. 302-321.

D’Aveni, R. (1995), Hypercompetition, Paris, Vuibert.

Revue internationale P.M.E., vol. 14, n 2, 2001

(C) 2001 - Presses de l'Université du Québec

Édifice Le Delta I, 2875, boul. Laurier, bureau 450, Sainte-Foy, Québec G1V 2M2 • Tél. : (418) 657-4399 - www.puq.uquebec.ca

Tiré de : Revue internationale P.M.E., vol. $14, \mathrm{n}^{\circ} 2$, sous la direction de Pierre-André Julien. 
DESS, G.C. et R.B. RoBINSON (1984), «Measuring organizational performance in the absence of objective measures : the case of the privately-held firm and conglomerate business unit », Strategic Management Journal, vol. 5, n 3, p. 265-274.

EVRARD, Y, B. Pras et E. RouX (1993), Market, études et recherches en marketing, Paris, Nathan.

Fombrum, C.J. et A. GinsBerG (1990), «Shifting gears : enabling change in corporate aggressiveness », Strategic Management Journal, vol. 11, n 5, p. 291-308.

FundenberG, D. et J. Tirole (1986), «A "signal-jaming" theory of predation », Rand Journal of Economics, vol. 17, p. 366-376.

GARDA, R. et M. MARN (1994), «Comment échapper à la guerre des prix », L'Expansion Management Review, n ${ }^{\circ}$ 72, p. 6-13.

Guillotreau, P. et F. LE Roy (1999), « Les comportements concurrentiels comme objet d'étude », Les Cahiers de l'Artemis, $\mathrm{n}^{\circ} 1$, Nantes, Presses de l'Université de Nantes.

GuNDLACH, G. T. (1990), «Predatory practices in competitive interaction: legal limits and antitrust considerations », Journal of Public Policy and Marketing, vol. 9, p. 02129-153.

Gupta, A.K. et V. GovindarajAn (1984), «Business unit strategy, managerial characteristics and business unit effectiveness at strategy implementation », Academy of Management Journal, vol. 27, $\mathrm{n}^{\circ}$ 1, p. 25-41.

HinTHORNE, T. (1996), «Predatory capitalism, pragmatism, and legal positivism in the airlines industry », Strategic Management Journal, vol. 17, nº 4, p. 251-270.

JACQUEMIN, A. (1985), Sélection et pouvoir dans la nouvelle économie industrielle, Paris, Economica; Louvain-la-Neuve, Cabay.

JULIEN, P.-A. (1990), «Notes de lecture: vers une typologie multicritère des P.M.E. », Revue internationale PME, vol 3, nos 3-4, p. 411-426.

Julien, P.-A. et M. MARChESNAY (1989), La petite entreprise, Paris, Vuibert.

KotLer, P. (1988), Marketing Management, Englewood Cliffs, NJ, Prentice-Hall.

LAWLESS, M.W. et L.K. FINCH (1989), « Choice and determinism : a test of Hrebiniak and Joyce framework on strategy-environment fit», Strategic Management Journal, vol. $10, \mathrm{n}^{\mathrm{o}} 4$, p. 351-365.

LE RoY, F. (1996), «L'agressivité concurrentielle comme objet de recherche », Actes des $X_{I I I}{ }^{e s}$ Journées nationales des IAE, tome 2, École supérieure universitaire de Toulouse, Toulouse, p. 395-408.

LE Roy, F. (1998), «Dynamique de la concurrence et cession d'activité : le cas de la cession de Cassegrain (Compagnie Saupiquet) au Groupe Bonduelle», Revue Finance Contrôle - Stratégie, vol. 1, no 1, p. 125-143.

LEVY, D.T. (1989), «Predation, firm-specific assets and diversification », The Journal of Industrial Economics, vol. 38, n 2, p. 227-233.

LUMPKIN, G.T. et G.G. DESS (1996), « Clarifying the entrepreneurial orientation construct and linking it to performance », Academy of Management Review, vol. 21, $\mathrm{n}^{\circ} 1$, p. $135-172$.

Revue internationale P.M.E., vol. 14, n 2, 2001

(C) 2001 - Presses de l'Université du Québec

Édifice Le Delta I, 2875, boul. Laurier, bureau 450, Sainte-Foy, Québec G1V 2M2 • Tél. : (418) 657-4399 - www.puq.uquebec.ca

Tiré de : Revue internationale P.M.E., vol. $14, \mathrm{n}^{\circ} 2$, sous la direction de Pierre-André Julien. 
MCMillan, I. (1980), « How business strategists can use guerrilla warfare tactics », Journal of Business Strategy, vol. 1, n 3, p. 63-65.

MiLes, R.E. et C.C. SNOw (1978), Organizational Strategy, Structure and Process, New York, McGraw-Hill.

ORDOVER, J.A. et R.D. WILLIG (1981), « An economic definition of predation : pricing and product innovation », Yale Law Journal, vol. 91, p. 8-53.

Ordover, J.A. et G. SAlONER (1989), Handbook of Industrial Organization, dans R. Schmalense et R.D. Willig, vol. 1, Amsterdam, Elsevier Science, B.V., p. 545-596.

Porac, J.F., H. Thomas, F. Wilson, D. PATON et A. KAnfer (1995), « Rivalry and the industry models of Scottish knitwear producers », Administrative Science Quarterly, vol. 40 , p. 203-277

PORTER, M. (1982), Choix stratégiques et concurrence, Paris, Economica.

PotTER, D.V. (1994), «Rare mettle: gold and silver strategies to succeed in hostile markets », California Management Review, vol. 34, n 1, p. 65-80.

RoBInson, W.T. (1988), « Marketing mix reactions to entry », Marketing Science, vol. 7, $\mathrm{n}^{\circ} 1$, p. $368-385$.

SCHERRER, F.M. (1980), Industrial Market Structure and Economic Performance, Boston, Rand McNally.

SCHERRER, F.M. et D. Ross (1990), Industrial Market Structure and Economic Performance, Boston, Houghton Mifflin.

Sharma, S., R.M. DURAND et O. GUR-ArIE (1981), «Identification and analysis of moderator variables », Journal of Marketing Research, vol. 18, no 3, p. 291-300.

SMith, K.G., C.M. GRIMM et M.J. GANNON (1993), Dynamics of Competitive Strategy, Londres, Sage Publications.

TIROLE, J. (1988), The Theory of Industrial Organization, Cambridge, MIT Press.

VENKATARAMAN, N. (1989), «The concept of fit in strategy research: toward verbal and statistical correspondence », Academy of Management Review, vol. 14, n 3, p. 423-444.

Williams, M.L., M.-H. TSA. et D. DAY (1991), «Intangible assets, entry strategies, and venture success in industrial markets », Journal of Business Venturing, vol. 16, $\mathrm{n}^{\circ} 5$, p. 315-333.

WiLLIAMSON, O.E. (1977), «Predatory pricing: a strategic and welfare analysis », Yale Law Journal, vol. 87, no 7, p. 284-340.

YounG, G., K.G. SMITH et C.M. GRIMM (1996), « “Austrian” and industrial organization perspectives on firm-level competitive activity and performance», Organization Science, vol. 7, no 3, p. 243-254.

ZhARA, S.A. et J.G. Covin (1993), «Business strategy, technology policy and firm performance », Strategic Management Journal, vol. 14, n 5, p. 451-478.

Revue internationale P.M.E., vol. 14, n 2, 2001

(C) 2001 - Presses de l'Université du Québec

Édifice Le Delta I, 2875, boul. Laurier, bureau 450, Sainte-Foy, Québec G1V 2M2 • Tél. : (418) 657-4399 - www.puq.uquebec.ca

Tiré de : Revue internationale P.M.E., vol. 14, $\mathrm{n}^{\circ} 2$, sous la direction de Pierre-André Julien. 